\title{
Environmental assessment of cytotoxic drugs in healthcare settings: protocol for a systematic review and meta-analysis
}

\author{
Laila Al Alawi ${ }^{1}$, Elpidoforos S. Soteriades ${ }^{1,2}$, Marilia Silva Paulo ${ }^{1,3}$, Linda Östlundh ${ }^{4}$, Michal Grivna',
} Fatima Al Maskari ${ }^{1,5}$ and Rami H. Al-Rifai ${ }^{1,5^{*}}$ (D)

\begin{abstract}
Background: Occupational exposure to cytotoxic drugs is associated with various unfavorable health outcomes. This protocol reports a methodology for a systematic review and meta-analysis that aims to systematically review the published literature and quantify the level of environmental contamination of healthcare settings with cytotoxic drugs.

Methods: This protocol is developed in accordance with the Preferred Reporting Items for Systematic Reviews and Meta-Analyses Protocol-2015 (PRISMA-P) guidelines. Six electronic databases (PubMed, Web of Science, Scopus, Cochrane Library, CINAHL, and EMBASE) will be searched with no restrictions on publication period. Eligible studies will be identified and data will be extracted using a predefined data extraction form by at least two independent reviewers following best practice. Eligible studies should report calculated or calculable estimates on the proportion of positive samples tested for cytotoxic drugs and/or estimates on the concentration of the cytotoxic drug(s) in the tested samples. Risk of bias (RoB) will be assessed by using the RoB in Studies estimating Prevalence of Exposure to Occupational risk factors (RoB-SPEO) tool, which developed by the World Health Organization (WHO) and International Labour Organization (ILO) for environmental and occupational health systematic reviews. The randomeffects model will be used to perform meta-analyses.

Discussion: Occupational exposure to cytotoxic drugs is associated with short- and long-term adverse health outcomes. Following this protocol, the review to be carried out will be the first to fill an evidence gap on the environmental contamination of healthcare settings with cytotoxic drugs. The findings of this review will help in the understanding of the risk of occupational exposure of healthcare workers to cytotoxic drugs and facilitate the identification of priority areas for specific interventions.
\end{abstract}

Ethics and dissemination: The systematic review methodology does not require ethics approval due to the nature of the study design. The results of the systematic review will be published in a peer-reviewed journal and will be publicly available.

Systematic review registration: PROSPERO CRD42020162780, dated July 14, 2020

Keywords: Cytotoxic drugs, Environmental assessment, Systematic review, Meta-analysis

\footnotetext{
* Correspondence: rrifai@uaeu.ac.ae

'Institute of Public Health, College of Medicine and Health Sciences, United Arab Emirates University, P.O. Box 17666, Al Ain, United Arab Emirates

${ }^{5}$ Zayed Center for Health Sciences, United Arab Emirates University, Al Ain, United Arab Emirates

Full list of author information is available at the end of the article
}

(c) The Author(s). 2020 Open Access This article is licensed under a Creative Commons Attribution 4.0 International License, which permits use, sharing, adaptation, distribution and reproduction in any medium or format, as long as you give appropriate credit to the original author(s) and the source, provide a link to the Creative Commons licence, and indicate if changes were made. The images or other third party material in this article are included in the article's Creative Commons licence, unless indicated otherwise in a credit line to the material. If material is not included in the article's Creative Commons licence and your intended use is not permitted by statutory regulation or exceeds the permitted use, you will need to obtain permission directly from the copyright holder. To view a copy of this licence, visit http://creativecommons.org/licenses/by/4.0/ The Creative Commons Public Domain Dedication waiver (http://creativecommons.org/publicdomain/zero/1.0/) applies to the data made available in this article, unless otherwise stated in a credit line to the data. 


\section{Background}

Hazardous drugs, as defined by the National Institute for Occupational Safety and Health (NIOSH), are the drugs that associated with genotoxicity, carcinogenicity, teratogenicity, reproductive toxicity, organ toxicity, or other developmental toxicity [1]. Cytotoxic drugs, also known as anti-neoplastic, chemotherapeutic, or hazardous drugs [2], are defined as a group of medicines designed to treat cancer through disrupting the cell cycle and destroy cells that grow in a rapid and uncontrolled manner [3]. They have extensively been used in the treatment of cancer patients and this has led to occupational hazards associated with exposure of healthcare professionals to such drugs leading to different adverse effects including spontaneous abortion [4], infertility [5, 6], premature labor, developmental and behavioral abnormalities among children of exposed workers, and potential cancer development [6]. Furthermore, several studies have reported widespread contamination of working areas which leads to healthcare workers' exposure to these hazardous drugs [7, 8].

Potential routes of occupational exposure may include dermal absorption, ingestion, and inhalation [1]. While levels of such exposures are usually much lower than those administered to cancer patients [4], occupational exposure likely involves a concurrent exposure to more than one cytotoxic drugs or several combined exposures, and it occurs more frequently over a much longer period of time leading to mixed cumulative exposures [9]. Furthermore, occupational exposures are often unrecognized due to lack of systematic environmental assessment and biomonitoring programs in oncology departments [10].

A recent systematic review conducted by the National Toxicology Program (NTP) USA 2019 [9] evaluated the literature on occupational exposure to cytotoxic drugs and its associated adverse health effects in humans. This systematic review also summarized the prevalence and levels of cytotoxic drugs detected in the workplace. According to this review, detectable levels of cytotoxic drugs continue to be identified in the workplace environment [9]. However, the risk of bias ( $\mathrm{RoB})$ of the reviewed studies and evaluation of confidence in the body of evidence were not reported. In addition, the review did not quantify the levels of environmental contamination of the workplace with cytotoxic drugs [9].

Several individual studies have been conducted on the cytotoxic drugs contamination in healthcare settings. These individual studies do not however assess the worldwide burden of cytotoxic drugs contamination in healthcare settings. Furthermore, the evidence is lacking on pooled estimates of the levels of healthcare setting contamination in order to provide national, international, and global figures. Therefore, the proposed systematic review and meta-analysis following this protocol aims to (1) systematically review the published literature, (2) estimate the pooled prevalence of environmental samples positive to cytotoxic drugs, and (3) estimate the weighted mean concentration of cytotoxic drugs, in the environmental samples collected from different healthcare settings around the world.

\section{Methods and data synthesis}

The review to be carried out following this protocol will be reported in accordance to the Preferred Reporting Items for Systematic Reviews and Meta-analyses (PRIS MA) guidelines [11].

\section{Outcome}

The main outcome measure in our study will be the pooled prevalence of positive samples of cytotoxic drugs in the tested environmental samples, and the mean concentration of cytotoxic drugs in environmental samples collected from healthcare settings.

\section{Protocol design and registration}

The development of this protocol is in accordance with the Preferred Reporting Items for Systematic Reviews and Meta-analyses Protocol (PRISMA-P) guidelines (S1 Table) [12]. The protocol is registered (ID CRD42020162780) on The International Prospective Register of Systematic Reviews (PROSPERO). Registration reduces duplication of reviews and provides transparency in the review process, with the aim of minimizing reporting bias. In the S2 Table, we provide an indicative International Agency for Research on Cancer (IARC) classification of cytotoxic drugs that have been adopted and will be used in our planned review $[13,14]$.

\section{Eligibility criteria}

We will include studies conducted in healthcare settings with no restrictions on publication or study period. All studies including samples from settings involved in the preparation, transport, administration, and waste disposal of cytotoxic drugs, will be considered. We will also consider healthcare settings in which these settings are exposed to cytotoxic drugs as participants with regard to outcomes such as surface contamination and aerosol contamination. Eligible studies should report quantitative data on the prevalence of positive samples and/or on the concentration of the detected cytotoxic drugs in the positive samples. A positive sample would be defined as a sample with cytotoxic drugs above the level of detection limit of cytotoxic drugs [15]. Studies reporting on calculated prevalence or those presenting data that would allow us to calculate the prevalence or concentration of cytotoxic drugs in the collected environmental samples will be included. No restrictions on study design 
will be applied. We will include only articles reported in the English language.

Studies will be excluded if are not conducted in healthcare settings such as those conducted in university laboratories, drug manufacturing companies and/or veterinaries. Studies that do not provide quantitative information on the prevalence or the concentration of positive environmental samples of cytotoxic drug residues will be not be deemed eligible for inclusion.

\section{Search strategy and searching sources}

A comprehensive systematic search in PubMed, Web of Science (Core Collection), Scopus, Cochrane Library, CINAHL, and Embase and in sources for grey literature will be conducted. The search strategy will be developed by the project team under the guidance of an experienced librarian. PubMed and PubMed's MeSH will be used to systematically develop a comprehensive search string. All search terms will be searched in a combination of title, abstract, and $\mathrm{MeSH} / \mathrm{Thesaurus}$ (when available) to ensure the best possible information retrieval. A filter for the English language will be applied. All publication years and publication types will be included. A detailed search log with transparent and reproducible search stings, results, and search variation notes for all included databases and sources for grey literature will be developed and reported. A preliminary search strategy conducted in PubMed is available in the S3 Table. Hand searching of the reference lists for studies that might have been potentially missed will be conducted.

\section{Study selection}

All citations identified through the literature search will be imported into the systematic review software "Covidence" [16] for deduplication and blinded screening. Titles and abstracts of the retrieved citations will be screened to exclude all ineligible studies against the pre-set inclusion criteria. Full texts of the eligible and potentially eligible studies will be thoroughly assessed for eligibility by at least two independent reviewers. Disagreements will be resolved through discussion and consensus after consulting a third reviewer whenever necessary. The corresponding authors of eligible articles will be contacted for clarification whenever needed. We will record all reasons for the exclusion and report the study selection process using the PRISMA flow diagram [11].

\section{Data extraction}

For studies found eligible to be included in the systematic review, relevant data will be extracted into a predefined data extraction form, which will first be piloted using five eligible studies. Data will be extracted by at least two reviewers. Data to be extracted from each eligible study will include baseline and methodological data. We will extract information related to the authors' names, publication year, country, studied cytotoxic drugs, sample size, sampling locations, sampling year(s), analytical tool, the sensitivity of contaminant measurements [limit of detection (LOD) or limit of quantitation (LOQ)] [17], number of tested samples, number of positive samples, and mean concentration of the tested cytotoxic drugs in the tested samples. A list of variables to be extracted from eligible studies are provided in S4 Table.

\section{Quality and risk of bias assessment}

At least two reviewers will independently evaluate and assess the methodological quality of the eligible studies. If required, the authors of the studies will be contacted to request missing or additional data for an explanation. Disagreements between the reviewers will be resolved by discourse. The results will be reported in narrative form and summarized in tables.

The risk of bias (RoB) of the included studies will be assessed using the RoB in Studies estimating Prevalence of Exposure to Occupational risk factors (RoB-SPEO) tool developed by the World Health Organization (WHO) and the International Labour Organization (ILO) for studies of the prevalence of exposure to occupational risk factors [18]. We will assess the RoB on the levels of each individual study and the entire body of evidence overall. We will resolve any disagreements by discussion. The RoB will be assessed according to the following domains: (i) selection bias, (ii) performance bias, (iii) misclassification bias, (iv) conflict of interest, and (v) other biases. Categorization of bias will be "low," "probably low," "probably high," "high," or "not applicable."

\section{Quality of evidence}

The quality of evidence of the included studies will be assessed using the quality and strength of evidence ratings proposed by the Navigation Guide as a framework. We will decrease, or not, the quality level of the body of evidence based on the (i) RoB across studies, (ii) indirectness of the evidence, (iii) inconsistency, (iv) imprecision, and (v) publication bias $[19,20]$. We will grade the evidence, using the three Navigation Guide quality of evidence ratings: "high," "moderate," and "low" [20]. Within each of the relevant reasons for downgrading, we will rate any concern per reason as "none," "serious," or "very serious."

\section{Synthesis of evidence: meta-analysis Pooled weighted measures}

We will provide a quantitative synthesis approach to report the prevalence of cytotoxic drugs contamination in environmental samples collected from healthcare 
settings. To estimate the pooled prevalence, we will conduct a meta-analysis using a random-effects model to estimate the pooled prevalence of positive samples for the tested cytotoxic contamination. We will use the metaprop command to perform a meta-analysis of the prevalence estimates [21]. To estimate the mean concentration of cytotoxic drugs in the tested environmental samples in healthcare settings, we will conduct a meta-analysis using a random-effects model. We will use the metan command to perform meta-analysis of cytotoxic drugs concentration in the tested samples. The pooled measures will be weighted using the inverse variance method [22]. For each pooled estimate and its 95\% confidence interval $(\mathrm{CI})$, a forest plot will be created to show the estimated overall weighted prevalence/ weighted mean concentration and its corresponding 95\% CI for each study following the Cochrane guidelines [23].

Heterogeneity between the studies in effect measures will be assessed using both the $Q$-test statistic and the $I$ squared $\left(I^{2}\right)$ statistic. We will consider a $Q$-test with a $p$ -value $<0.05$ and $I^{2}$ statistic of $>50 \%$ as indicative of substantial heterogeneity.

\section{Subgroup analyses}

Depending on data availability, we expect to conduct subgroup analyses stratifying by geographical regions, time periods, type of the tested cytotoxic drug, study settings, sample locations, and quality/bias assessment classifications. We will also conduct subgroup analyses based on study quality.

\section{Discussion}

The extensive use of cytotoxic drugs in the treatment of cancer patients has led to occupational hazards associated with exposure of healthcare professionals to such drugs. Exposure to cytotoxic drugs leads to different adverse health effects such as spontaneous abortion, infertility, premature labor, and developmental and behavioral abnormalities. Systematically compiling and summarizing primary studies as well as providing comprehensive estimates on the burden of cytotoxic drugs in healthcare settings is still lacking in the literature. This protocol reduces the possibility of duplication, minimizes possible biases, and allows peer-review using the proposed methods to conduct a high-quality systematic review and metaanalysis. The systematic review to be produced following this protocol, as far as we know, is the first systematic review and meta-analysis to provide systematic evidence on the levels of environmental contamination of the healthcare settings with cytotoxic drugs. The results of the systematic review will be published in a peer-reviewed journal and will be publicly available. The findings will also be disseminated electronically and in printed versions.

\section{Supplementary information}

Supplementary information accompanies this paper at https://doi.org/10. 1186/s13643-020-01494-4.

Additional file 1: Table S1. PRISMA-P (Preferred Reporting Items for Systematic review and Meta-Analysis Protocols) 2015 checklist.

Additional file 2: Table S2. IARC classification of cytotoxic drugs.

Additional file 3: Table S1. Search specifications: All terms are searched in the fields for "Title" and "Abstract" and in "MeSH" when available. No filters or limitations applied

Additional file 4: Table S1. List of string and numerical variables to be extracted from eligible studies.

\section{Abbreviations}

Cl: Confidence interval; IARC: International Agency for Research on Cancer;

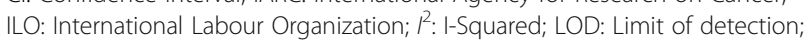
LOQ: Limit of quantitation; NIOSH: National Institute for Occupational Safety and Health; NTP: National Toxicology Program; PRISMA-P: Preferred Reporting Items for Systematic Reviews and Meta-analyses Protocol; PRIS MA: Preferred Reporting Items for Systematic Reviews and Meta-analyses; PROSPERO: International Prospective Register of Systematic Review; RoBSPEO: Risk of Bias in Studies estimating Prevalence of Exposure to Occupational risk factors; WHO: World Health Organization

\section{Acknowledgements}

None.

\section{Authors' contributions}

LA, ESS, and RHA conceived of the idea for the study. All authors contributed to the design of the study. RHA designed the analysis plan. LÖ developed the full search strategy for each database. LA wrote the first draft of the manuscript. All authors contributed to subsequent versions of the manuscript and critically revised the protocol. All authors contributed to the final version of the manuscript. All authors read and approved of the final manuscript.

Funding

No specific funding has been allocated for this proposed work

\section{Availability of data and materials}

Data sharing is not applicable to this article as no datasets were generated or analyzed during the current study.

\section{Ethics approval and consent to participate}

The systematic review methodology does not require ethics approval due to the nature of the study design. Patients and the public will not be involved in this study.

\section{Consent for publication}

Not applicable. No individuals involved in this study.

\section{Competing interests}

The authors declare that they have no competing interests.

\section{Author details}

${ }^{1}$ Institute of Public Health, College of Medicine and Health Sciences, United Arab Emirates University, P.O. Box 17666, Al Ain, United Arab Emirates. ${ }^{2}$ Department of Environmental Health, Environmental and Occupational Medicine and Epidemiology (EOME), Harvard School of Public Health, Boston, MA, USA. ${ }^{3}$ Global Health and Tropical Medicine, Instituto de Higiene e Medicina Tropical, Universidade Nova de Lisboa, Lisbon, Portugal. ${ }^{4}$ National Medical Library, College of Medicine and Health Sciences, United Arab Emirates University, Al Ain, United Arab Emirates. ${ }^{5}$ Zayed Center for Health Sciences, United Arab Emirates University, Al Ain, United Arab Emirates. 
Received: 14 May 2020 Accepted: 23 September 2020

Published online: 19 October 2020

\section{References}

1. National Institute for Occupational Safety and Health (2016) NIOSH Workplace Safety and Health Topic: NIOSH list of antineoplastic and other hazardous drugs in healthcare settings. Available at https://www.cdc.gov/ niosh/docs/2016-161/default.html . Accessed 13 Aug 2020.

2. National Institute for Occupational Safety and Health (2019). Reproductive health and the workplace. Available at https://www.cdc.gov/niosh/topics/ repro/antineoplastic.html. Accessed 21 Aug 2020.

3. European Biosafety Network. 2020. Preventing occupational exposure to cytotoxic drugs. Available at https://www.europeanbiosafetynetwork.eu/ preventing-occupational-exposure-to-cytotoxic-drugs/. Accessed 24 Aug. 2020

4. Lawson CC, Rocheleau CM, Whelan EA, Lividoti Hibert EN, Grajewski B, Spiegelman D, et al. Occupational exposures among nurses and risk of spontaneous abortion. Am J Obstet Gynecol. 2012;206(4):327 e1-8.

5. Connor TH, Lawson CC, Polovich M, McDiarmid MA. Reproductive health risks associated with occupational exposures to antineoplastic drugs in health care settings: a review of the evidence. J Occup Environ Med. 2014 56(9):901-10.

6. National Institute for Occupational Safety and Health (2020). Hazardous drug exposure in healthcare. Available at https://www.cdc.gov/niosh/topics/ hazdrug/default.html. Accessed 21 Aug 2020.

7. Sessink PJ, Leclercq GM, Wouters DM, Halbardier L, Hammad C, Kassoul N. Environmental contamination, product contamination and workers exposure using a robotic system for antineoplastic drug preparation. J Oncol Pharm Pract. 2015;21(2):118-27.

8. Muller-Ramirez C, Squibb K, McDiarmid M. Measuring extent of surface contamination produced by the handling of antineoplastic drugs in low- to middle-income country oncology health care settings. Arch Environ Occup Health. 2017;72(5):289-98.

9. National Institute of Environmental Health Sciences. NTP monograph on the systematic review of occupational exposure to cancer chemotherapy agents and adverse health outcomes. 2019 https://ntp.niehs.nih.gov/ntp/ohat/ oeccaaho/mgraph/occchemo_final20190300_508.pdf.

10. Occupational Safety and Health Administration (OSHA). Controlling occupational exposure to hazardous drugs. Available at https://www.osha. gov/SLTC/hazardousdrugs/controlling_occex_hazardousdrugs.html. Accessed 13 Aug 2020.

11. Liberati A, Altman DG, Tetzlaff J, Mulrow C, Gotzsche PC, loannidis JP, et al. The PRISMA statement for reporting systematic reviews and meta-analyses of studies that evaluate health care interventions: explanation and elaboration. PLoS Med. 2009;6(7):e1000100.

12. Moher D, Shamseer L, Clarke M, Ghersi D, Liberati A, Petticrew M, et al. Preferred reporting items for systematic review and meta-analysis protocols (PRISMA-P) 2015 statement. Syst Rev. 2015;4:1.

13. International Agency for Research on Cancer (IARC). Agents classified by the IARC monographs, Voumes 1-125. World Health Organization, 2019.

14. Fransman W. Antineoplastic drugs: Occupational exposure and health risks. Utrecht University Repository. Ridderprint offsetdrukkerij BV, Ridderkerk, the Netherlands; 2006:12. Available from: https://dspace.library.uu.nl/handle/1 874/12854.

15. Chauchat L, Tanguay C, Caron NJ, Gagne S, Labreche F, Bussieres JF. Surface contamination with ten antineoplastic drugs in 83 Canadian centers. J Oncol Pharm Pract. 2019;25(5):1089-98.

16. Covidence systematic review software. Melbourne, Australia: Veritas Health Inovation Ltd. Available at https://www.covidence.org.

17. Connor TH, Zock MD, Snow AH. Surface wipe sampling for antineoplastic (chemotherapy) and other hazardous drug residue in healthcare settings: methodology and recommendations. J Occup Environ Hyg. 2016;13(9):658-67.

18. Pega F, Norris SL, Backes C, Bero LA, Descatha A, Gagliardi D, et al. RoBSPEO: a tool for assessing risk of bias in studies estimating the prevalence of exposure to occupational risk factors from the WHO/ILO joint estimates of the work-related burden of disease and injury. Environ Int. 2020;135:105039.

19. Woodruff TJ, Sutton P. The navigation guide systematic review methodology: a rigorous and transparent method for translating environmental health science into better health outcomes. Environ Health Perspect. 2014;122(10):1007-14

20. J. Lam, P. Sutton, A.M. Padula, M.D. Cabana, E. Koustas, H.M. Vesterinen, et al. Applying the Navigation Guide Systematic review methodology case study
\#6: association between formaldehyde exposure and asthma: a systematic review of the evidence: protocol. University of California at San Francisco, San Francisco, CA (2016); 2016.

21. Nyaga VN, Arbyn M, Aerts M. Metaprop: a Stata command to perform metaanalysis of binomial data. Arch Public Health. 2014;72(1):39.

22. Freeman MF, Tukey JW. Transformations related to the angular and the square root. Ann Math Stat. 1950;21:607-11.

23. Higgins JPT, Thomas J, Chandler J, Cumpston M, Li T, Page MJ, Welch VA. Cochrane Handbook for Systematic Reviews of Interventions version 6.0. 2019; Available from: www.training.cochrane.org/handbook.

\section{Publisher's Note}

Springer Nature remains neutral with regard to jurisdictional claims in published maps and institutional affiliations.
Ready to submit your research? Choose BMC and benefit from:

- fast, convenient online submission

- thorough peer review by experienced researchers in your field

- rapid publication on acceptance

- support for research data, including large and complex data types

- gold Open Access which fosters wider collaboration and increased citations

- maximum visibility for your research: over $100 \mathrm{M}$ website views per year

At BMC, research is always in progress.

Learn more biomedcentral.com/submissions 